\title{
Insight Mechanistic of Hydroxychloroquine on Ventricular Myocytes and Tissue in COVID-19 and Common Comorbidities: An In-silico Perspective
}

\author{
Ponnuraj Kirthi Priya ${ }^{1}$ and Srinivasan Jayaraman ${ }^{1}$ \\ ${ }^{1}$ Tata Consultancy Services Ltd
}

June 16, 2020

\begin{abstract}
Objective: To investigate HCQ interaction mechanistic under COVID-19 with and without pro-arrhythmic comorbidities such as Long QT syndrome (LQTS1 \& 2), and hypokalemia in (a) three types of cardiomyocytes (b) ventricular tissue and its effects when excited with premature beats (PBs) to understand the possibility of arrhythmogenesis. Methods and Results: A 2D transmural anisotropic ventricular tissue model consisting of endocardial, mid myocardial and epicardial myocytes are configured for mild and severe COVID-19, comorbid, and HCQ conditions. Results show that along with QT interval reduction, low amplitude and/or inversion T-wave occurred in mild and severe COVID-19 respectively. In contrast, under LQTS1 and mild hypokalemia leads to notched T-waves, and HCQ inclusion increases the QT interval and T-peak in all mild infections. Severe COVID-19 causes inverted T-waves and shorten QT-interval in all comorbidities except in LQTS2, where biphasic T-waves are observed. Arrhythmogenesis, reentry is created only on the addition of mild hypokalemia while ST elevation is observed in the presence of moderate and severe hypokalemia. When treated with HCQ, no significant impact occurred. Conclusion: In-silico ventricular model indicates, HCQ has an insignificant effect on COVID-19 without and with comorbidities, except in the combination of mild COVID-19 with moderate hypokalemia condition and severe COVID-19 with mild hypokalemia where it initiated a re-entrant arrhythmia. These results could help guide COVID-19 management
\end{abstract}

\section{Hosted file}

Hydroxy_Manuscript.pdf available at https://authorea.com/users/334030/articles/460103insight-mechanistic-of-hydroxychloroquine-on-ventricular-myocytes-and-tissue-in-covid19-and-common-comorbidities-an-in-silico-perspective 